\title{
Epidemiología del síndrome de abstinencia alcohólica. Mortalidad y factores de mal pronóstico
}

\author{
R. PUERTA LOURO, E. OTERO ANTÓN, V. LORENZO ZÚÑIGA \\ Servicio de Medicina Interna. Complexo Hospitalario Universitario de Santiago. \\ Santiago de Compostela. A Coruña
}

\begin{abstract}
EPIDEMIOLOGY OF ALCOHOL WITHDRAWAL SYNDROME. MORTALITY AND FACTORS OF POOR PROGNOSTIC
\end{abstract}

\section{RESUMEN}

Introducción: El alcoholismo y la aparición del síndrome de abstinencia alcohólica (SAA) condicionan un peor pronóstico en los pacientes que lo presentan.

Métodos: Se recogieron las características epidemiológicas, evolución y mortalidad de todos los casos diagnosticados de SAA en el Complexo Hospitalario de Santiago durante los años 1997 al 2002.

Resultados: Se identificaron 924 casos con una mortalidad del 2,4\% ( $1,6 \%$ en Medicina Interna). La mortalidad se asoció con mayor edad (57 años \pm 15 vs. $49 \pm 13, \mathrm{p}<0,005)$, y con los diagnósticos de cirrosis hepática $(6,2$ vs. $1,8 \%, \mathrm{p}<0,005)$, bacteriemia $(10 v s .1,8 \%, \mathrm{p}<0,001)$ e infección respiratoria $(9,6$ vs. $1,8 \%, \mathrm{p}<0,001)$, con una mortalidad menor cuando el SAA fue diagnóstico secundario $(1,2$ vs. $4,2 \%$, p < $0,005)$. En el análisis multivariante se asociaron a mayor mortalidad la edad (OR 1,03), la cirrosis hepática (OR 3,4), la bacteriemia $(\mathrm{OR} 4,5)$ y la infección respiratoria (OR 3,6).

Conclusión: La mortalidad asociada al síndrome de abstinencia podría beneficiarse de la centralización del tratamiento en el Servicio de Medicina Interna.

PALABRAS CLAVE: Alcoholismo. Abstinencia. Mortalidad.

\section{ABSTRACT}

Introduction: Patients with alcoholism and alcohol withdrawal syndrome (AWS) have a worse prognostic.

Methods: We have performed a retrospective analysis of the hospital discharges in with diagnosis include AWS from 1 of January of 1997 to the 31 of December of 2002.

Results: We identified 924 hospital stays with 2.4\% of mortality (1.6\% in Internal Medicine). Mortality is associated with greater age (57 years $\pm 15 v s .49 \pm 13, p<0.005$ ), with the diagnostic of hepatic cirrhosis (6.2 vs. $1.8 \%, p<0.005)$, bacteraemia $(10 \mathrm{vs.} 1.8 \%, p<0.001)$ and respiratory infection ( $9.6 \mathrm{vs} .1 .8 \%, p<0,001)$, with a lower mortality when AWS was secondary diagnosis (1.2 vs. $4.2 \%, p<0.005)$. In multivariant analysis were associated with more mortality age (OR 1.03), hepatic cirrhosis (OR 3.4), bacteriemia (OR 4.5) and respiratory infection (OR 3.6).

Conclusion: Alcohol withdrawal syndrome mortality could to benefit from treatment in an Internal Medicine Service.

KEY WORDS: Alcoholism. Alcohol Withdrawal. Mortality.

Puerta Louro R, Otero Antón E, Lorenzo Zúñiga V. Epidemiología del síndrome de abstinencia alcohólica. Mortalidad y factores de mal pronóstico. An Med Interna (Madrid) 2006; 23: 307-309.

\section{INTRODUCCIÓN}

El alcoholismo es un problema de salud frecuente que afecta a un 5-10\% de la población, siendo esta prevalencia mayor entre los pacientes que ingresan en un hospital, hasta un 50\% en Servicios como Traumatología o Psiquiatría (1-3). La aparición de sintomatología relacionada con la falta de consumo de alcohol en estos pacientes, y que definimos como síndrome de abstinencia alcohólica (SAA), debería ser por lo tanto frecuente. La prevalencia exacta del SAA es difícil de establecer debido a la utilización generalizada de tratamientos preventivos (4).
El SAA es un cuadro potencialmente grave que en la década de 1960 tenía una mortalidad del 15\% y desde entonces es menor del $2 \%$ posiblemente relacionado con un tratamiento más adecuado (5). El ingreso en la Unidad de Cuidados Intensivos es más frecuente y con mayor mortalidad en los pacientes alcohólicos $(6,7)$.

La población gallega tiene además unas características peculiares respecto a otras comunidades de España y otros países, con un mayor consumo y frecuencia de bebedores de grandes cantidades de alcohol, relacionándose este consumo con factores socioculturales como la edad joven, profesión de labrador y bajo estatus socioeconómico (8).

Trabajo aceptado: 7 de febrero de 2006 
El objetivo del presente estudio fue estudiar la prevalencia del SAA entre los pacientes ingresados en el Complexo Hospitalario Universitario de Santiago (CHUS) y sus características epidemiológicas.

\section{MATERIAL Y MÉTODOS}

Revisamos los ingresos en el CHUS entre el 1 de enero de 1997 y 31 de diciembre de 2002 entre cuyos diagnósticos figuraba el SAA. Recogimos los datos referentes a edad, sexo, tiempo de estancia, servicio de ingreso, estancias en diferentes servicios, procesos o enfermedades de los que fueron diagnosticados, así como la evolución. Analizamos la influencia en la mortalidad de la edad, el sexo, servicio de ingreso, estancia en UCI, patologías asociadas y diagnóstico principal.

\section{RESULTADOS}

Identificamos 924 casos diagnosticados de SAA, con una incidencia anual de 154 casos (rango 137-168) y la estancia mediana fue de 12 días (rango 1-179). La edad media de los pacientes fue de $50 \pm 13$ años, y el $85 \%$ eran varones.

El 53\% ingresó inicialmente en Medicina Interna, el 14\% en Psiquiatría, el 9\% en Digestivo, el 9\% en la Unidad de Cuidados Intensivos y el $15 \%$ en el resto de los servicios médicos y quirúrgicos. Precisaron a lo largo de su estancia hospitalaria ingreso en Medicina Interna un 56\%.

Ingresaron inicialmente o fueron trasladados a la Unidad de Cuidados Intensivos un 18,5\% (Fig. 1). La necesidad de traslado a la UCI fue mayor entre los pacientes ingresados en los Servicios Quirúrgicos y en Cardiología. En este último el $80 \%$ de sus pacientes ingresaron en la UCI, en probable relación con la patología asociada ya que de los 10 casos, 6 presentaban IAM, uno disección aórtica y otro insuficiencia cardiaca.

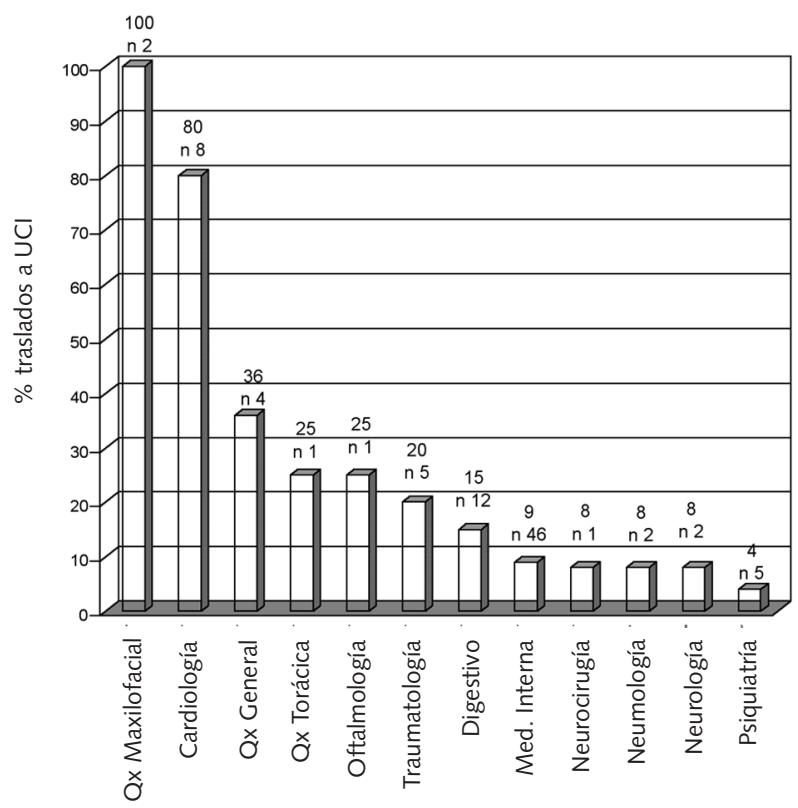

Fig. 1. Porcentaje de traslados a UCl según el servicio.
Los diagnósticos asociados al SAA se detallan en la figura 2. El SAA fue el diagnóstico principal en 566 casos $(61 \%)$. Destacan como diagnósticos más frecuentes la cirrosis $(12 \%)$, la hepatitis alcohólica $(9 \%)$ y la infección respiratoria $(8 \%)$. Otros diagnósticos presentes son accidente (7\%), anemia $(7 \%)$, bacteriemia $(6 \%)$, enfermedad pulmonar obstructiva crónica $(6 \%)$, trombopenia $(5,5 \%)$, fractura $(5,5 \%)$, infección del tracto urinario $(5 \%)$, hemorragia digestiva alta $(5 \%)$ y varices esofágicas $(5 \%)$.

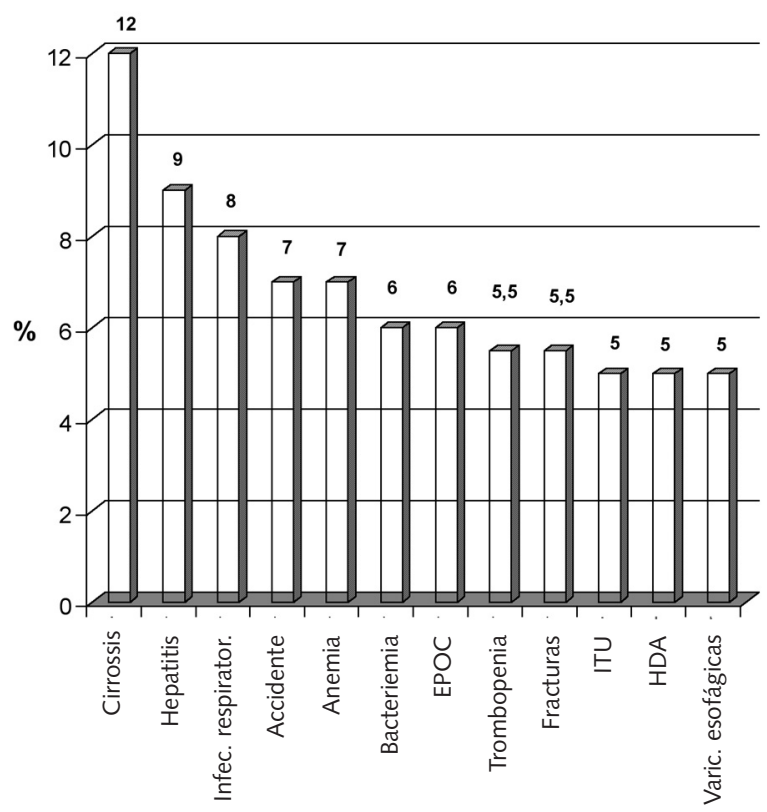

Fig. 2. Otros diagnósticos presentes en los pacientes con síndrome de abstinencia alcohólica.

EPOC: enfermedad pulmonar obstructiva crónica; ITU: infección del tracto urinario; HDA: hemorragia digestiva alta.

La mortalidad global fue un 2,4\% siendo menor (1,6\%) en Medicina Interna que en otros servicios como Cardiología (20\%), Neurología (12,5\%), Neurocirugía (6,7\%), UCI (6\%), Neumología (4\%) o Digestivo (2,5\%).

La mortalidad se asoció con mayor edad (57 años \pm 15 vs. $49 \pm 13, \mathrm{p}<0,005)$, y con los diagnósticos de cirrosis hepática $(6,2$ vs. 1,8\%, p < 0,005), bacteriemia $(10$ vs. 1,8\%, p < 0,001) e infección respiratoria $(9,6$ vs. $1,8 \%, \mathrm{p}<0,001)$, con una mortalidad menor cuando el SAA fue diagnóstico secundario (1,2 vs. $4,2 \%, \mathrm{p}<0,005$. En el análisis multivariante se asociaron a mayor mortalidad la edad (OR 1,03), la cirrosis hepática (OR $3,4)$, la bacteriemia (OR 4,5) y la infección respiratoria (OR 3,6).

\section{DISCUSIÓN}

En la década de los 80 en nuestro hospital la prevalencia del SAA era de un $2,44 \%$ (9).

El SAA fue un diagnóstico frecuente de los ingresos en MIR, un 4,38\% (492 de 11.234 ingresados en estos años), y en más de la mitad de los casos constituyó el motivo fundamental de ingreso hospitalario. Es casi 6 veces más frecuente en varones, y entre la $4^{\mathrm{a}}$ y $5^{\mathrm{a}}$ década de la vida. 
La mayoría de los pacientes ingresan en Medicina Interna o bien son trasladados a este servicio desde otros, como la UCI o los servicios quirúrgicos. Existen diversos protocolos de actuación o directrices para el manejo de estos pacientes (10-12).

Durante el ingreso es frecuente la necesidad de traslado a UCI, sobre todo cuando el SAA acontece en un paciente ingresado por otro motivo en un servicio quirúrgico, con la excepción del Servicio de Cardiología ligado a la gravedad de la patología relacionada y condicionando además el mal pronóstico de estos pacientes.

Es frecuente la asociación con hepatitis alcohólica, cirrosis hepática e infecciones respiratorias, siendo además estos dos últimos diagnósticos factores de mal pronóstico.

La mortalidad es baja, similar a la publicada en la literatura y es menor entre los pacientes ingresados en el servicio de
Medicina Interna. Esto es probablemente debido a la amplia experiencia en el manejo de esta patología y a la aplicación de un protocolo específico de manejo y que además la frecuencia de patologías médicas asociadas, como aparición de fiebre y complicaciones infecciosas (infecciones respiratorias y bacteriemia) $(13,14)$ y de alteraciones hidroelectrolíticas que se pueden producir en estos pacientes como la deshidratación (resultado de sudoración, hipertermia, vómitos y taquipnea), la hipopotasemia (por pérdidas renales y extrarrenales), la hipomagnesemia (que puede predisponer a convulsiones), e hipofosfatemia (que puede presentarse debido a la malnutrición) (15), hacen que el manejo y pronóstico de este cuadro pudiera beneficiarse de la centralización de todos los casos en Medicina Interna. Son necesarios otros estudios que evalúen la eficacia de la aplicación de un protocolo de actuación en todos los pacientes diagnosticados de SAA.

\section{Bibliografía}

1. Schorling JB, Buchsbaum DG. Screening for alcohol and drug abuse. Med Clin North Am 1997; 81: 845-65.

2. Saitz R., O’Malley S.S. Pharmacotherapies for alcohol abuse. Withdrawal and Treatment. Med Clin North Am 1997; 81: 881-907.

3. Kraemer KL, Conigliaro J, Saitz R. Managing Alcohol Withdrawal in the Elderly. Drugs Aging 1999; 14: 409-25.

4. Batel P. Alcohol withdrawal syndrome in the hospital. Rev Prat 1999; 49: 387-90.

5. Williams D, McBride AJ. The drug treatment of alcohol withdrawal symptoms: a systematic review. Alcohol Alcohol 1998; 33: 103-15.

6. Delgado-Rodríguez M, et al. Alcohol drinking as a predictor of intensive care and hospital mortality in general surgery: a prospective study. Addiction 2003; 98: 611-6.

7. Powell A. H. Alcohol Withdrawal in critical care. Alcohol withdrawal in critical care. Dimens Crit Care Nurs 1999; 18: 24-8.

8. Mateos R, Paramo M, Carrera I, Rodríguez-Lopez A. Alcohol consumption in a southern European region (Galicia, Spain). Subst Use Misuse 2002; 37: 1957-76.

9. Gippini Pérez A, Rodriguez Lopez I, Torre Carballada A, Tomé y Martínez de Rituerto S, Barrio Gómez E. Alcohol withdrawal syndrome at

the internal medicine department of a general hospital; epidemiology and hospital costs. An Med Interna (Madrid) 1990; 7: 171-3.

10. Mayo-Smith MF. Pharmacological management of alcohol withdrawal: a meta-analysis and evidence-base practice guideline: American Society of addiction Medicine Working Group on Pharmacological Management of Alcohol Withdrawal. JAMA 1997; 278: 144-51.

11. Kosten TR, O'Connor PG. Management of Drug and Alcohol Withdrawal. N Engl J Med 2003; 348: 1786-95.

12. Mayo-Smith MF, Beecher LH, Fischer TL, Gorelick DA, Guillaume JL, Hill A, et al. Working Group on the Management of Alcohol Withdrawal Delirium, Practice Guidelines Committee, American Society of Addiction Medicine. Management of alcohol withdrawal delirium. An evidencebased practice guideline. Arch Intern Med 2004; 164: 1405-12.

13. Wrenn KD, Larson S. The febrile alcoholic in the emergency department. Am J Emerg Med 1991; 9: 57-60.

14. Rose HD, Golbert TM, Sanz CJ, Leitschuh TH. Feber during acute alcohol withdrawal. Am J Med Sci 1970; 260: 112-21.

15. Stasiukyniene V. Blood plasma potassium, sodium and magnesium levels in chronic alcoholism during alcohol withdrawal. Medicina (Kaunas) 2002; 3: 892-5. 